\title{
EFECTIVIDAD DE ANESTESIA DE PLEXO CERVICAL SUPERFICIAL PREOPERATORIO PARA CONTROL DE DOLOR POSTOPERATORIO EN CIRUGÍA DE TIROIDES*
}

\author{
Drs. Nicolás Ávalos J. ${ }^{1,2}$, Carolina Cabrera Sch. ${ }^{3}$, Irini Semertzakis P. ${ }^{3}$, Silvia Schmied P. ${ }^{3}$ \\ 1 Departamento de Cirugía de Cabeza y Cuello. Hospital Clínico de la Fuerza Aérea de Chile, \\ Universidad de Valparaíso. \\ 2 Instituto Chileno de Cabeza y Cuello. \\ 3 Departamento de Anestesiología, Hospital Clínico de la Fuerza Aérea de Chile, Universidad de Valparaíso. \\ Santiago, Chile.
}

\section{Abstract \\ Analgesic effects of bilateral superficial cervical plexus block with Bupivacaine after thyroidectomy}

Background: Bilateral superficial cervical plexus block is a simple non-invasive technique that can be used as preventive analgesia in the perioperative period of thyroidectomy. Aim: to assess the analgesic effects of the technique during the postoperative period of thyroidectomy. Material and Methods: Patients with indication of total thyroidectomy, with a low operative risk according to the American Society of Anesthesiology, were studied. All were operated with general anesthesia using Fentanyl, Propofol, Vecuronium and Isoflurane. Patients were randomly and blindly assigned to superficial cervical plexus block using Bupivacaine $0.25 \%$ or to a placebo injection. Postoperative pain, need for analgesics and patient satisfaction were assessed. Results: Thirty five patients aged $55+15$ years $(28$ women) were studied. Mean use of morphine in the Bupivacaine and placebo groups was 5.8 and $10.4 \mathrm{mg}$, respectively $(\mathrm{p}<0.05)$. All and $75 \%$ of patients in placebo and Bupivacaine groups respectively, required morphine in some moment of the postoperative period. Nausea and vomiting was observed in 55 and $93 \%$ of patients in Bupivacaine and placebo groups, respectively $(\mathrm{p}<0.05)$. Mean Ondansetron use was 2.1 and $4.6 \mathrm{mg}$ in Bupivacaine and placebo groups, respectively $(\mathrm{p}<0.05)$. No significant differences between groups were observed for patient satisfaction. Conclusions: Bilateral superficial cervical plexus block with Bupivacaine reduces analgesic use in the postoperative period of thyroidectomy.

Key words: Cervical plexus block, analgesia, thyroidectomy.

\section{Resumen}

Introducción: La cirugía de la glándula tiroides es reconocida como un procedimiento que produce un dolor leve a moderado. El bloqueo bilateral de plexo cervical superficial es una técnica simple, poco invasiva,

*Recibido el 31 de marzo de 2014 y aceptado para publicación el 21 de abril de 2014.

No hubo apoyo financiero. Los autores no refieren conflictos de interés.

Correspondencia: Dr. Nicolás Ávalos J.

contacto@cabezaycuello.cl 
que pudiera ser beneficioso en estos pacientes como modelo de analgesia preventiva. Objetivos: Evaluar la calidad de la analgesia del postoperatorio de la cirugía de tiroides, con el uso de bloqueo bilateral del plexo cervical superficial. Material y Métodos: Se estudiaron pacientes ASA I y II propuestos para cirugía de tiroides bajo anestesia general. En todos los pacientes se hizo anestesia general balanceada con Fentanyl, Propofol, Vecuronio e Isoflurano. En forma aleatoria y ciega se asignaron los pacientes en 2 grupos: grupo A, Bupivacaína $0,25 \% 20 \mathrm{ml}$ y grupo B placebo. Se evaluó características demográficas, dolor postoperatorio, necesidad de analgésicos de rescate, náuseas, vómitos y satisfacción del paciente. Resultados: 35 pacientes, 7 hombres y 28 mujeres. Con edad promedio de 55,3 $\pm 14,8$ ASA I y 27 ASA II. Quince pacientes en grupo A y 20 pacientes grupo B. Comparables en variables demográficas. El uso promedio de morfina fue de 10,4 mg en grupo B y $5,8 \mathrm{mg}$ en grupo A $(\mathrm{p}<0,05)$. En el grupo Bupivacaína hubo un $25 \%$ de pacientes que no usaron morfina en ningún momento del postoperatorio, mientras que en el grupo placebo todos usaron morfina en algún momento de su postoperatorio. Al estudiar la presencia de náuseas y/o vómitos postoperatorios, se encuentran en el 93\% de los pacientes del grupo B versus el 55\% de los pacientes del grupo A $(\mathrm{p}<0,05)$. El consumo de fármacos de rescate también fue superior en el grupo placebo (B) siendo este en promedio de $4,6 \mathrm{mg}$ de ondasentrón por paciente en $24 \mathrm{~h}$ versus $2,1 \mathrm{mg}$ por paciente en el grupo $\mathrm{A}(\mathrm{p}<0,05)$. Al evaluar la satisfacción de los pacientes, esta no presentó diferencias estadísticamente significativas entre ambos grupos. Conclusiones: El bloqueo bilateral del plexo cervical superficial es un coadyuvante en la analgesia de la cirugía de tiroides, que permite disminuir el uso de opioides y secundariamente la aparición de náuseas y vómitos, mejorando la calidad y seguridad del postoperatorio de esta cirugía.

Palabras clave: Tiroidectomía, analgesia preventiva, bloqueo plexo cervical superficial, analgesia post operatoria.

\section{Introducción}

La cirugía tiroidea es un procedimiento frecuente. $\mathrm{Su}$ dolor postoperatorio varía entre leve a moderado. Muchos reportes muestran niveles de dolor en Escala Visual Análoga de 6 en el 90\% de casos, con necesidad de opioides como droga de rescate ${ }^{1,2}$. El manejo del dolor agudo postoperatorio ha demostrado ser una medida eficiente para mejorar el confort del postoperatorio y disminuir la respuesta de stress frente a la injuria quirúrgica. La disminución del dolor postoperatorio también es una medida eficiente para alcanzar un mejor nivel de satisfacción de los pacientes Por estas razones es una preocupación permanente de buscar el mejor esquema de analgesia $^{3}$. La analgesia preventiva, es decir, el inicio de la terapia analgésica antes de que se produzca la noxa quirúrgica, ha demostrado su utilidad en diferentes tipos de cirugías. Su efecto consiste en la disminución de los valores máximos en la escala de dolor y una importante disminución en el consumo de morfina postoperatoria por diversos mecanismos ${ }^{4}$. Particularmente en el caso de una tiroidectomía, el uso de opioides aumenta la incidencia de náuseas y vómitos, ambos efectos muy indeseados. Los vómitos pueden aumentar el riesgo de hematoma a tensión de cuello, la complicación más temida durante el postoperatorio de una tiroidectomía 5 .

Con el objetivo de realizar analgesia preventiva en una tiroidectomía se plantea el uso de un bloqueo de plexo cervical superficial. Es un procedimiento sencillo y de bajas complicaciones, que podría con- tribuir a disminuir el dolor agudo postoperatorio y el uso de morfina postoperatoria.

La hipótesis de trabajo fue demostrar la utilidad del bloqueo de plexo cervical superficial pre-incisión quirúrgica, para disminuir el dolor agudo en el postoperatorio de tiroides y el consumo de morfina como modelo de analgesia preventiva.

\section{Material y Método}

Luego de aprobación del estudio por el comité de ética del hospital y firma de consentimiento informado por cada paciente, se estudiaron pacientes adultos ASA I y II sometidos a tiroidectomía total. Ellos fueron randomizados en dos grupos: Grupo $A$ : bloqueo de plexo cervical con bupivacaína al $0,25 \%, 10$ cc a cada lado del cuello. Grupo B: bloqueo plexo cervical con solución fisiológica $10 \mathrm{cc}$ por cada lado del cuello. De manera prospectiva, randomizada y doble ciego fueron incluidos de manera consecutiva aquellos enfermos propuestos para una tiroidectomía total. Para lograr el doble ciego, la randomización del paciente y la preparación de la jeringa con solución a inyectar fue preparada por enfermera de anestesia fuera del pabellón. Esta enfermera luego no participó en los cuidados post operatorios inmediatos. $\mathrm{Ni}$ el anestesista, ni el cirujano, ni el paciente conocían en ese momento la solución a administrar. Ciego se abrió sólo en la tabulación de datos. Se excluyeron aquellos pacientes que tenían una cirugía previa cervical, enfermos con terapia de 
anticoagulación y enfermos portadores de demencia de algún grado.

El paciente ingresó al quirófano donde fue monitorizado de manera no invasiva. Luego se instaló un vía endovenosa periférica con una solución de Ringer lactato. La inducción anestésica se realizó utilizando una mezcla entre fentanyl, propofol y un relajante muscular no depolarizante. Para la mantención anestésica se utilizó isofluorano. Todos los pacientes fueron intubados por vía orotraqueal y recibieron $8 \mathrm{mg}$ de dexametasona. Al finalizar la cirugía el paciente recibe $4 \mathrm{mg}$ de ondasentrón de profilaxis de náuseas y vómitos.

El bloqueo cervical superficial se realizó de manera estándar a todos los casos luego de la inducción anestésica, usando como referencia el músculo esternocleidomastideo y la vena yugular externa. En el punto de cruce entre ambos, y posterior a la vena, se inyectó la solución con aguja de $21 \mathrm{G}$ levemente curvada para realizar punción paralela a la mesa operatoria distribuyendo la dosis en forma de abanico. De esta forma aseguramos el bloqueo del punto de Erb y ramas más caudales del plexo cervical. Posterior al bloqueo se confeccionó los campos quirúrgicos y se inició la cervicotomía.

Luego de su cirugía todos los enfermos fueron trasladados a una unidad de cuidado de postoperatorio. Todos los pacientes recibieron la misma modalidad de analgesia post operatoria, con ketoprofeno endovenoso por horario (100 mg cada $8 \mathrm{~h}$ ). Se instaló bomba PCA (Patient Controlled Analgesia) de morfina a todos los enfermos y se registró cantidad de droga usada, efectos adversos (náuseas, vómitos, somnolencia, retención urinaria) y necesidad de droga de rescate para vómitos (ondasentron). Se registraron complicaciones postoperatorias tales como hematoma de cuello, hematoma en sitio de punción, seroma, anestesia del frénico y anestesia de plexo braquial. Se midió cada dos horas Escala Visual Análoga (EVA) para medir el dolor. Se aplicó un cuestionario de satisfacción al alta con- sistente en cuestionario simple con escala de 1 a 5 , realizado por enfermera no relacionada con atención del paciente.

Los resultados se analizaron con paquete estadístico STATA 10.0 , utilizando $\chi^{2}$ para variables continuas y test " $\mathrm{t}$ " Student considerando como significativo un $\mathrm{p}$-value menor a 0,05 .

\section{Resultados}

Se estudiaron 35 pacientes, 7 hombres y $28 \mathrm{mu}-$ jeres. Con edad promedio de 55,3 \pm 14 años. Ocho ASA I y 27 ASA II. Veinte pacientes en grupo A y 15 pacientes grupo B. Las características demográficas de los pacientes no tuvieron diferencia estadística entre ambos grupos, lo que permitió su comparación (Tabla 1).

El uso promedio de morfina fue de $5,8 \mathrm{mg}$ en grupo A y 10,4 mg en grupo B. El uso promedio de morfina endovenosa por PCA fue significativamente menor en el grupo estudio con bloqueo de plexo cervical con bupivacaína $(p<0,05)$. Incluso, en el grupo estudio un $25 \%$ de los pacientes no requirió morfina de rescate, mientras en el grupo placebo todos usaron morfina en algún momento (Tabla 1).

$\mathrm{Al}$ estudiar la presencia de náuseas y/o vómitos postoperatorios hay en el $93 \%$ de los pacientes del grupo B (placebo) versus el 55\% de los enfermos del grupo A. La presencia de náuseas y vómitos en el grupo estudio con bloqueo con bupivacaína fue significativamente menor $(p<0,05)$. El consumo de fármacos de rescate también fue superior en el grupo placebo (B) siendo este en promedio de 4,6 $\mathrm{mg}$ de ondasentrón por paciente en $24 \mathrm{~h}$ versus $2,1 \mathrm{mg}$ por paciente en el grupo A; es decir que los requerimientos de droga antiemética como el ondasentrón fueron menores en el grupo estudio $(\mathrm{p}<0,05)$ (Tabla 1$)$.

No se registró ninguna complicación atribuible al uso del bloqueo del plexo cervical superficial. No hubo hematomas de cuello en la serie, ni seromas.

Tabla 1. Resultados

\begin{tabular}{|lccc|}
\hline & Grupo A (bupivacaína) & Grupo B (placebo) & \\
Pacientes & 20 & 15 & $\mathrm{p}<0,05$ \\
Uso morfina promedio & $5,8 \mathrm{mg} / 24 \mathrm{~h}$ & $10,4 \mathrm{mg} / 24 \mathrm{~h}$ & $\mathrm{p}<0,05$ \\
Uso morfina en pacientes (\%) & $75 \%$ usó & $100 \%$ sí usó & $\mathrm{p}<0,05$ \\
Presencia náuseas y vómitos & $55 \%$ & $93 \%$ & Sin dif. \\
\hline Uso ondasentrón promedio & $2,1 \mathrm{mg} / 24 \mathrm{~h}$ & $4,6 \mathrm{mg} / 24 \mathrm{~h}$ & No \\
Complicaciones & No & Bueno & \\
Nivel satisfacción & Bueno & & \\
\hline
\end{tabular}


Ambos grupos tuvieron un buen control de la analgesia postoperatoria y valores de EVA bajo 5 en todo momento.

A nivel de satisfacción de usuario, ambos grupos fueron dados de alta con buena satisfacción frente al control del dolor y comodidad en el post operatorio, valor promedio de 4,4 puntos (escala de 1 a 5 ), sin diferencia significativa entre los grupos.

\section{Discusión}

En este estudio de investigación se demostró que el bloqueo de plexo cervical superficial previo a tiroidectomía disminuyó los requerimientos de morfina postoperatoria, así como la incidencia de náuseas y vómitos. Destaca que un $25 \%$ de los pacientes en el grupo estudio no requirió morfina, demostrando la efectividad del bloqueo preventivo preoperatorio. De esta manera podemos brindar una analgesia post operatoria eficiente, con un procedimiento sencillo y de bajo costo. Estos resultados son consistentes con otros similares ${ }^{6,7}$.

En el grupo de estudio la presencia de náuseas y vómitos y los requerimientos de uso de ondasentrón fueron menores. El que la disminución de ambas variables sea significativa ratifica la observación de la efectividad del bloqueo de plexo cervical preventivo. La disminución de la presencia de náuseas y vómitos en parte se debe a la menor dosis de morfina requerida para controlar el dolor. Todos los pacientes recibieron una dosis inicial de ondasentrón como profilaxis de náuseas y vómitos; y el uso de dexametasona en tiroidectomía ya está establecido como parte de este cuidado ${ }^{8,9}$. Al disminuir las náuseas y vómitos el paciente no sólo está más cómodo, sino que podría, al menos teóricamente, disminuir la incidencia del número de hematomas a tensión de cuello, que es la complicación más temida. Esto no se pudo demostrar por contar el trabajo con 35 pacientes y ser la tasa de hematoma de cuello menor a $0,5 \%$ anual en nuestro equipo. La disminución de náuseas y vómitos es un objetivo fundamental en toda unidad de post anestesia y un factor muy importante en la satisfacción de los pacientes.

Este es un modelo de analgesia preventiva que mostró resultados positivos, como también hay otros mecanismos que pueden ser atendidos con el uso de COX2 preoperatorio y de gabapentina ${ }^{4,10,11}$.

En forma indirecta hay disminución de costos al disminuir los requerimientos de analgésicos y de antieméticos, además el paciente puede ser dado de alta en $12 \mathrm{~h}$, a diferencia del paciente sintomático que debe permanecer un día más en el hospital. El tema de los costos no fue evaluado directamente en este estudio.
El tema de analgesia y tiroidectomía tiene muchos aspectos que continúan en evaluación, con medidas del tipo de analgesia preventiva buscamos elaborar un protocolo de tiroidectomía ambulatoria en el corto plazo ${ }^{12-15}$.

\section{Referencias}

1. Motamed C, Merle JC, Yakhou L, Combes X, Dumerat $\mathrm{M}$, Vodinh $\mathrm{J}$, et al. Intraoperative i.v. morphine reduces pain scores and length of stay in the post anaesthetic care unit after thyroidectomy. Br J Anaesth. 2004;93:306-7.

2. Motamed C, Merle JC, Yakhou L, Combes X, Vodinh J, Kouyoumoudjian C, et al. Postoperative pain scores and analgesic requirements after thyroid surgery: comparison of three intraoperative opioid regimens. Int J Med Sci. 2006;3:11-3.

3. Gehling M, Arndt C, Eberhart LH, Koch T, Krüger T, Wulf H. Postoperative analgesia with parecoxib, acetaminophen, and the combination of both: a randomized, double-blind, placebo-controlled trial in patients undergoing thyroid surgery. $\mathrm{Br} \mathrm{J}$ Anaesth. 2010;104:761-7.

4. Katz J, Clarke H, Seltzer Z. Preventive Analgesia: Quo Vadimus? Anesth Analg 2011;113:242-53.

5. Feroci F, Rettori M, Borrelli A, Lenzi E, Ottaviano A, Scatizzi M. Dexamethasone prophylaxis before thyroidectomy to reduce postoperative nausea, pain, and vocal dysfunction: a randomized clinical controlled trial. Head Neck 2011;33:840-6.

6. Andrieu G, Amrouni H, Robin E, Carnaille B, Wattier JM, Pattou F, et al. Analgesic efficacy of bilateral superficial cervical plexus block administered before thyroid surgery under general anaesthesia. Br J Anaesth. 2007;99:561-6.

7. Cai HD, Lin CZ, Yu CX, Lin XZ. Bilateral superficial cervical plexus block reduces postoperative nausea and vomiting and early postoperative pain after thyroidectomy. J Int Med Res. 2012;40:1390-8.

8. Chen CC, Siddiqui FJ, Chen TL, Chan ES, Tam KW. Dexamethasone for prevention of postoperative nausea and vomiting in patients undergoing thyroidectomy: meta-analysis of randomized controlled trials. World J Surg. 2012;36:61-8.

9. Zhou H, Xu H, Zhang J, Wang W, Wang Y, Hu Z. Combination of dexamethasone and tropisetron before thyroidectomy to alleviate postoperative nausea, vomiting, and pain: randomized controlled trial. World J Surg. 2012;36:1217-24.

10. Al-Mujadi H, A-Refai AR, Katzarov MG, Dehrab NA, Batra YK, Al-Qattan AR. Preemptive gabapentin reduces postoperative pain and opioid demand following thyroid surgery. Can J Anaesth. 2006;53:268-73.

11. Karamanlioğlu B, Arar C, Alagöl A, Colak A, Gemlik I, 
Süt N. Preoperative oral celecoxib versus preoperative oral rofecoxib for pain relief after thyroid surgery. Eur J Anaesthesiol. 2003;20:490-5.

12. Abdulla S, Eckhardt R, Netter U, Abdulla W. Efficacy of three IV non-opioid-analgesics on opioid consumption for postoperative pain relief after total thyroidectomy: a randomised, double-blind trial. Middle East J Anesthesiol. 2012;21:543-52.

13. Lee JH, Lee HK, Chun NH, So Y, Lim CY. The prophylactic effects of gabapentin on postoperative sore throat after thyroid surgery. Korean J Anesthesiol. 2013;64:138-42.

14. Lee SY, Lee WH, Lee EH, Han KC, Ko YK. The effects of paracetamol, ketorolac, and paracetamol plus morphine on pain control after thyroidectomy. Korean J Pain. 2010;23:124-30.

15. Smirnov G, Terävä M, Tuomilehto $H$, Hujala K, Seppänen M, Kokki H. Etoricoxib for pain management during thyroid surgery-a prospective, placebo-controlled study. Otolaryngol Head Neck Surg. 2008;138:92-7. 